01,11

\title{
Метастабильные дисперсные состояния, возникающие при распаде трехкомпонентного сплава
}

\author{
(C) И.К. Разумов ${ }^{1}$, Ю.Н. Горностырев ${ }^{1,2}$ \\ ${ }^{1}$ Институт фризики металлов им. М.Н. Михеева УрО РАН, \\ Екатеринбург, Россия \\ ${ }^{2}$ Уральский федеральный университет им. Б.Н. Ельцина, \\ Екатеринбург, Россия \\ E-mail: rik@imp.uran.ru
}

Поступила в Редакцию 6 мая 2019 г.

В окончательной редакции 16 июля 2019 г.

Принята к публикации 16 июля 2019 г.

Рассмотрены условия торможения роста выделений из метастабильного пересыщенного твердого раствора, и на стадии испарения-конденсации при спинодальном распаде трехкомпонентного сплава. Показано, что эффективным механизмом торможения распада является формирование „запирающей“ оболочки вокруг выделений, при условии, что в ней понижены растворимость или коэффициенты диффузии компонент сплава; сформулированы термодинамические и кинетические условия возникновения таких оболочек. Обсуждаются особенности механизмов торможения распада в конкретных сплавах.

Ключевые слова: спинодальный распад, трехкомпонентный сплав, дисперсные состояния.

DOI: 10.21883/FTT.2019.12.48580.474

\section{1. Введение}

В последние годы значительное внимание привлекают материалы, уникальные свойства которых достигаются благодаря формированию структурного состояния, содержащего наноразмерные выделения. Известными примерами являются мартенситные стареющие стали $[1,2]$, сплавы на основе алюминия [3], титана [4] и переходных металлов с композитной структурой [5]. Перспективным способом управления структурным состоянием является использование легирующих элементов, повышающих стабильность выделений [6-9]. Однако, физические механизмы, обеспечивающие устойчивость дисперсных состояний, недостаточно изучены. Существующие представления выделяют в качестве главных факторов упругие напряжения $[10,11]$, зависимость состава выделений от их размера, сегрегации примесей на межфазных границах $[12,13]$ и появление кинетически обусловленной оболочки вокруг выделений [14].

Влияние легирующего элемента на кинетику распада исследовалось в ряде работ на примере трехкомпонентного сплава. В работах $[15,16]$ было показано, что даже при выборе средней концентрации третьего компонента в области растворимости, межфазная граница может обогащаться им благодаря формированию особого состояния в условиях локального равновесия. Однако, исследования этого эффекта [16-18] на примере сплава $\mathrm{Fe}-\mathrm{Cu}-\mathrm{Ni}(\mathrm{Mn}, \mathrm{Al})$ показали, что обогащение границы выделений $\mathrm{Cu}$ примесями $\mathrm{Ni}(\mathrm{Mn}, \mathrm{Al})$ мало сказывается на кинетике роста выделений.

При нарушении когерентности решетки на межфазной границе, она становится подобна границе зерна, и создаются благоприятные условия для сегрегации на ней примесных атомов. В этом случае могут формироваться аномальные дисперсные состояния, с равновесным размером выделений, возрастающим при уменьшении средней концентрации примеси [12]. В работе [13] детально исследована стабилизация выделений $\mathrm{Mg}_{2} \mathrm{Sn}$ за счет сегрегации $\mathrm{Zn}$ на межфазной границе и определен их равновесный размер.

Важную роль в формировании дисперсной структуры играют не только термодинамические, но и кинетические факторы. Так в сплаве $\mathrm{Al}-\mathrm{Sc}-\mathrm{Zr}$ различие диффузионной подвижности $\mathrm{Zr}$ и $\mathrm{Sc}$ приводит к формированию кинетически обусловленной оболочки вокруг выделений $\mathrm{Al}_{3} \mathrm{Sc}_{x} \mathrm{Zr}_{1-x}$, которая блокирует огрубление микроструктуры [14]. Другая ситуация рассмотрена в работе [19], где проводилось моделирование распада бинарного сплава с введенными в начальное состояние неподвижными частицами третьей фазы, присутствие которых затормаживало распад. В работе [20] рассмотрен случай формирования выделений смешанного состава и показано, что распад замедляется, если одна из примесей имеет низкий коэффициент диффузии.

В данной работе мы рассматриваем распад в трехкомпонентном сплаве, уделяя особое внимание механизмам ограничения роста первичных, когерентных с матрицей выделений. Полученные результаты позволяют сформулировать условия, при которых формируется оболочка вокруг выделений и достигается стабилизация дисперсных состояний сплава.

\section{2. Формулировка модели}

В приближении среднего поля функционал свободной энергии Гинзбурга-Ландау [10] для трехкомпонентного 
$A-B-M$ сплава имеет вид

$$
F=\int\left\{f\left(c_{A}, c_{B}\right)+E_{\text {surf }}\right\} d \mathbf{r},
$$

где $f\left(c_{A}, c_{B}\right)$ - плотность свободной энергии, зависящая от локальных значений концентраций $c_{A}(\mathbf{r}), c_{B}(\mathbf{r})$, $E_{\text {surf }}$ - вклад в поверхностную энергию обусловленный градиентами концентраций; концентрации компонент связаны условием $c_{A}+c_{B}+c_{M}=1$. Плотность свободной энергии содержит вклады связанные с внутренней энергией и конфигурационной энтропией [21]:

$$
f\left(c_{A}, c_{B}\right)=g\left(c_{A}, c_{B}\right)+k T \sum_{\alpha} c_{\alpha} \ln c_{\alpha} .
$$

Плотность внутренней энергии имеет вид

$$
\begin{aligned}
& g\left(c_{A}, c_{B}\right)=\sum_{\alpha} E_{\alpha} c_{\alpha} \\
& =g_{0}+\varepsilon_{A} c_{A}+\varepsilon_{B} c_{B}+v_{A M} c_{A}^{2}+v_{B M} c_{B}^{2}+v c_{A} c_{B}
\end{aligned}
$$

где $g_{0}=$ const, $\varepsilon_{\alpha}-$ энергия растворения компонента $\alpha$ в матрице, $v_{\alpha \beta}-$ энергии смешения компонент $\alpha, \beta$; $v=v_{A M}+v_{B M}-v_{A B}$. При условии $v_{\alpha M}<0$ обеспечивается возможность распада с выделением компонент $\alpha=\{A, B\}$, в то время как энергия $v$ характеризует склонность атомов $A, B$ к формированию выделений смешанного состава $(v<0)$ или, наоборот, к распаду $(v>0)$. Последовательный вывод выражения (3) из конфигурационного гамильтонина трехкомпонентного сплава предполагает пренебрежение корреляциями в расположении атомов и плавное изменение концентраций на расстояниях порядка параметра решетки [22].

В общем случае $\varepsilon_{\alpha}$ различаются в разных фазах, что может быть учтено посредством концентрационной зависимости $\varepsilon_{\alpha}\left(c_{A} \cdot c_{B}\right)$. Опустим в (3) линейные вклады, которые не сказываются на термодинамике превращений, и перейдем к эффективным энергиям смешения, включающим в себя концентрационную зависимость величин $\varepsilon_{\alpha}, v_{\alpha M}, v_{A B}$ :

$$
\begin{aligned}
g\left(c_{A}, c_{B}\right)= & \tilde{v}_{A M}\left(c_{A}, c_{B}\right) c_{A}^{2}+\tilde{v}_{B M}\left(c_{A}, c_{B}\right) c_{B}^{2} \\
& +\tilde{v} c_{A} c_{B}\left(c_{A}, c_{B}\right) .
\end{aligned}
$$

Энергию $E_{\text {surf }}$ запишем в виде [23]:

$$
E_{\text {surf }}=\frac{R^{2}}{2}\left(\sigma_{A}\left(\nabla c_{A}\right)^{2}+\sigma_{B}\left(\nabla c_{B}\right)^{2}+\sigma_{A B}\left(\nabla c_{A} \nabla c_{B}\right) .\right)
$$

Здесь $R$ - параметр характеризующий ширину межфазной границы; $\sigma_{\alpha}-$ энергетические коэффициенты определяющие поверхностную энергию выделений сортов $A, B ; \sigma_{A B}-$ изменение поверхностной энергии при контакте выделений разных сортов. Если $\sigma_{A B}$ выше критического значения, энергия $E_{\text {surf }}$ становится отрицательной и следует ожидать реализации фазового перехода полного смачивания [24]. В общем случае энергии $\sigma_{\alpha}$ могут зависеть от локальных концентраций компонент сплава, $\sigma_{\alpha}=\sigma_{\alpha}^{(0)}+\sigma_{\alpha \beta} c_{\beta}+\cdot$, приводя к вкладам в функционал вида $c_{\beta}\left(\nabla c_{\alpha}\right)^{2}$, ответственным за сегрегацию примеси на межфазных границах [12]. В данной работе мы ограничимся анализом случая $E_{\text {surf }}>0$ и не будем учитывать в уравнении (5) градиентные вклады более высокого порядка.

Для описания эволюции концентраций используем уравнения непрерывности [21]:

$$
\frac{\partial c_{A(B)}}{\partial t}=-\nabla J_{A(B)}
$$

Потоки атомов $J_{A(B)}$ определяются линейной комбинацией градиентов химических потенциалов всех компонент, $J_{i}=-c_{i} M_{i j} \nabla\left(\delta F / \delta c_{j}\right)$, причем для недиагональных компонент матрицы подвижностей выполняются соотношения Онзагера, $M_{i j}=M_{j i}[21]$. Поскольку определяющий вклад обычно вносят диагональные элементы, примем $M_{i j}=M_{j i}=0$ при $i \neq j$, так что

$$
J_{A(B)}=-\frac{D_{A(B)}\left(c_{A}, c_{B}\right) c_{A(B)} c_{M}}{k T} \nabla\left(\frac{\delta F}{\delta c_{A(B)}}\right) .
$$

Коэффициенты диффузии компонент различаются в разных фазах, а на межфазной границе происходит их быстрое изменение, что можно учесть феноменологически:

$$
D_{\alpha}=D_{\alpha}^{(M)}+\left(D_{\alpha}^{(\beta)}-D_{\alpha}^{(M)}\right)\left[1+\operatorname{Exp}\left(\frac{c_{\beta}^{c r}-c_{\beta}}{\lambda}\right)\right]
$$

где $D_{\alpha}^{(M)}, D_{\alpha}^{(\beta)}$ - коэффициенты диффузии атомов сорта $\alpha=\{A, B\}$ в матрице и выделениях сорта $\beta$ соответственно, $c_{\beta}^{c r}-$ пороговая концентрация соответствующая появлению фазы $\beta$, малый параметр $\lambda \sim 0.01$. Коэффициенты самодиффузии для простоты примем равными коэффициентам диффузии в матрице, $D_{\alpha}^{(\alpha)} \approx D_{\alpha}^{(M)}$.

Анализ выражения (7) позволяет сделать общие выводы об условиях стабилизации выделений при распаде сплава. Термодинамически равновесное дисперсное состояние возникает, когда химические потенциалы компонент не зависят от координат $\left(\delta F / \delta c_{\alpha}=\right.$ const). Такое состояние может быть реализовано вследствие упругого взаимодействия выделений $[10,11]$ или в результате сегрегации примеси на межфазных границах $[12,13]$. Кинетический механизм стабилизации выделений реализуется, если становится мал множитель перед градиентом в уравнении (7), что достигается если выделение окружено оболочкой, в которой малы коэффициенты диффузии $\left(D_{A(B)} \approx 0\right)$ либо равновесная концентрация $\left(c_{A(B)} \approx 0\right)$ компонент сплава. Выполнения этих условий следует ожидать, когда оболочка, формирующаяся вокруг выделения, является новой фазой.

При численном решении уравнений (6) используем двухслойную явную конечно-разностную схему для квадратной 2D области, с безразмерным временем 
$\tau=t D_{A}^{(M)} / L^{2}$ и координатами $x / L(L-$ размер расчетной области). При анализе решения удобно использовать интегральную степень распада для компонента $A$

$$
S_{d e c}=\frac{1}{2 c_{A}^{0}\left(1-c_{A}^{0}\right) L^{d}} \int\left|c_{A}(\mathbf{r})-c_{A}^{0}\right| d \mathbf{r},
$$

где $d-$ размерность задачи, $c_{A}^{0}-$ средняя по образцу концентрация компонента $A, 0 \leq S_{d e c} \leq 1$. Для характеристики степени дисперсности выделений используем величину

$$
S_{d i s p}=\frac{R^{2}}{L^{d}} \int \sqrt{\left(\nabla c_{A}(\mathbf{r})\right)^{2}} d \mathbf{r}
$$

\section{3. Примесные оболочки вокруг выделений в двухфазных сплавах}

\section{1. Кинетически обусловленная оболочка}

Сначала рассмотрим рост одиночного выделения из метастабильного пересыщенного твердого раствора (фигуративная точка на фазовой диаграмме находится между бинодалью и спинодалью [21]) в простейшем случае, когда $\tilde{v}_{A M}<0, \tilde{v}_{B M}=\tilde{v}=0$. Пусть средняя концентрация примеси $c_{B}^{0}$ выше равновесного предела растворимости компонента $A$ в матрице $c_{A \_ \text {eqv }}^{(M)}$. Тогда компонент $B$ вытесняется из растущего выделения $A$ в матрицу. Если при этом скорость диффузии компонента $B$ в матрице мала $\left(D_{B}^{(M)} \ll D_{A}\right)$, а в объеме выделения достаточно велика $\left(D_{B}^{(A)} \sim D_{A}\right)$, на промежуточной стадии превращения вокруг выделения возникает оболочка с высокой концентрацией компонента $B$. Предполагая в этом разделе осевую симметрию задачи, при решении уравнения (6) мы используем цилиндрические координаты. Рассчитанные профили концентрации представлены на рис. 1, $a$. Прорастание выделения $A$ сквозь сформированную оболочку невозможно, так как при высокой концентрации компонента $B$ локально нарушены условия распада (фигуративная точка на фазовой диаграмме сплава смещена в область растворимости по компоненту $A$ ). Поэтому дальнейшая эволюция требует смещения либо растворения оболочки, что лимитируется низким коэффициентом $D_{B}^{(M)}$. В результате скорость распада существенно уменьшается (сравни кривые 1 и 2 на рис. $1, b$, построенные в отсутствие и при наличии оболочки).

\section{2. Обогащение межфазной границы примесью при взаимодействии компонент $\boldsymbol{A}$ и $B$}

Рассмотрим более общий случай $\tilde{v}_{A M}<0, \tilde{v}<0$, $\tilde{v}_{B M}=0$, при котором внутренняя энергия $g=\tilde{v}_{A M} c_{A}^{2}$ $+\tilde{v} c_{A} c_{B}$ понижается за счет кластеризации как атомов сорта $A$, так и атомов разных сортов $A, B$. Если $\tilde{v} / \tilde{v}_{A M}>1$, отношение равновесных концентраций компонента $B$ в матрице и объеме выделения
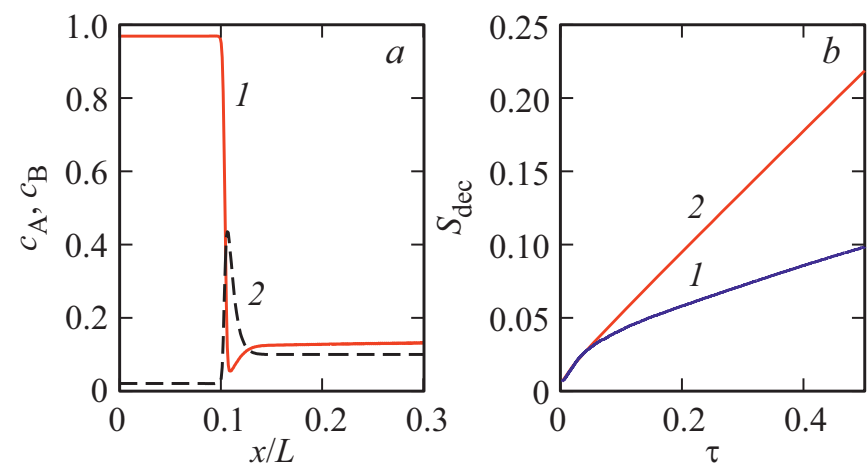

Рис. 1. $a-$ распределение концентраций компонент $A(1)$ и $B$ (2) в момент времени $\tau=2.65$ при $D_{B}^{(M)} / D_{A}=10^{-4}$, $D_{B}^{(A)} / D_{A}=3 ; \quad b \quad$ - эволюция степени распада при $D_{B}^{(M)} / D_{A}=10^{-4}, \quad D_{B}^{(A)} / D_{A}=3 \quad(1) ; \quad D_{B} / D_{A}=10^{-4} \quad$ (2). $\tilde{v}_{A M} / k T=-4, \quad \tilde{v}_{B M}=\tilde{v}=0, \quad c_{A}^{0}=0.15, \quad c_{B}^{0}=0.10$, $\sigma_{A(B)}(R / L)^{2} / k T=10^{-5}, \sigma_{A B}=0 ; x-$ расстояние до центра образца.
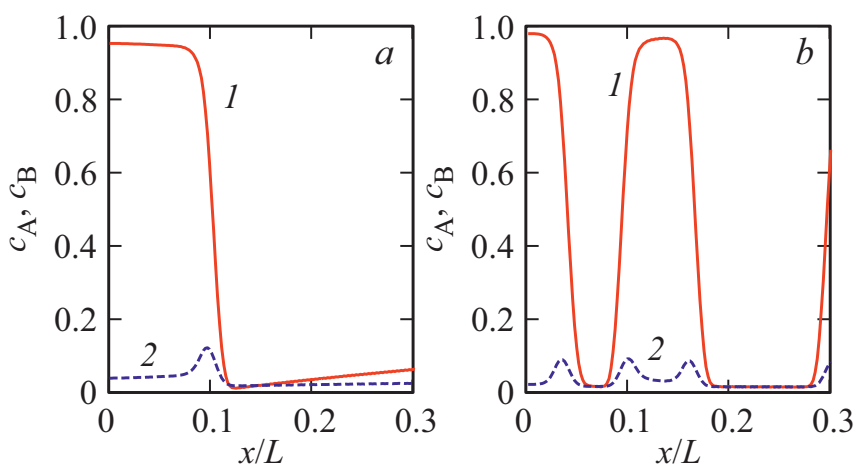

Рис. 2. Распределения компонент $A(1)$ и $B$ (2) в случае роста выделения из метастабильного пересыщенного раствора при $c_{A}^{0}=0.10, \tau=0.08(a)$; и при спинодальном распаде при $c_{A}^{0}=0.25, \tau=0.32(b) . c_{B}^{0}=0.03, D_{B} / D_{A}=1, \tilde{v}_{A M} / k T=-5$, $\tilde{v} / k T=-6, \tilde{v}_{B M}=0, \sigma_{A(B)}(R / L)^{2} / k T=4 \cdot 10^{-4}, \sigma_{A B}=0 ; x-$ расстояние до центра выделения.

$c_{B \_ \text {eqv }}^{(A)} / c_{B \_ \text {eqv }}^{(M)}>1$. При этом концентрация $c_{B}$ на межфазной границе демонстрирует необычное поведение, в разы превышая равновесные значения $c_{B \_ \text {eqv }}^{(A)}, c_{B \_ \text {eqv }}^{(M)}$. В результате на межфазной границе образуется оболочка, обогащенная компонентом $B$ (рис. 2,a). При понижении температуры или повышении концентрации $c_{A}^{0}$ фигуративная точка перемещается в область фазовой диаграммы ниже купола спинодали, и в сплаве реализуется спинодальный распад, в процессе которого также формируются выделения с межфазной границей обогащенной компонентом $B$ (рис. $2, b$ ).

Формирование такой оболочки находит простое объяснение. Из условия постоянства химического потенциала компонента $B$ на межфазной границе при достижении локального равновесия, в упрощающих предположениях $c_{A \_ \text {eqv }}(x) \gg c_{A \_ \text {eqv }}^{(M)}, c_{B \_ \text {eqv }}^{(M)} \ll 1$ легко получить выражение, связывающее равновесные концентрации примесей 


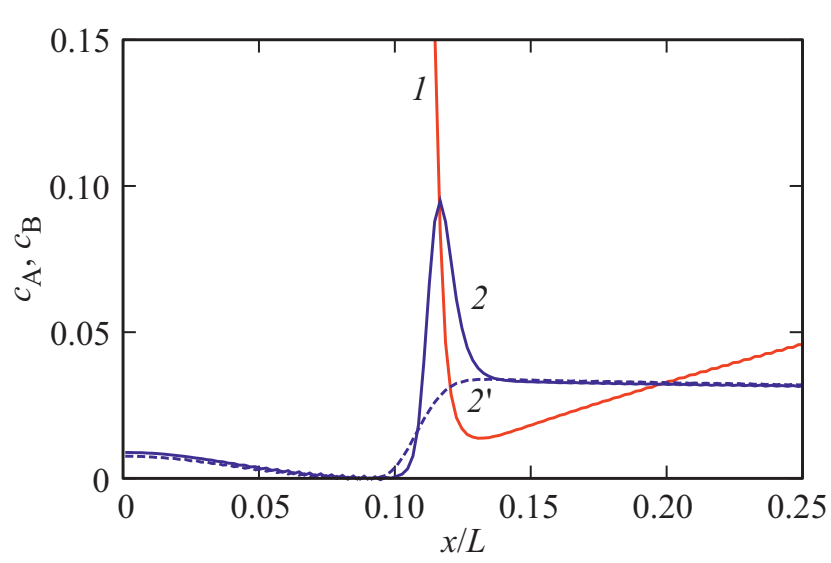

Рис. 3. Распределения компонент $A(1)$ и $B\left(2,2^{\prime}\right)$ при росте выделения из метастабильного пересыщенного раствора при $\tilde{v}=0, \sigma_{A B}(R / L)^{2} / k T=7 \cdot 10^{-4}(2), 0\left(2^{\prime}\right) ; \tau=0.10$, остальные параметры аналогичны рис. 2, $a$.

на межфазной границе:

$$
c_{B_{\_} e q v}\left(C_{A_{-} e q v}\right)=\frac{\left(1-c_{A_{\_} e q v}(x)\right) c_{B_{e} e q v}^{(M)}}{c_{B \_e q v}^{(M)}+\operatorname{Exp}\left[\frac{\tilde{v} c_{A_{\_} e q v}(x)}{k T}\right]} .
$$

Отсюда видно, что зависимость $c_{B_{-} e q v}\left(c_{A_{-} e q v}\right)$ немонотонна, так что при промежуточном значении концентрации $c_{A \_q q v}$ (т.е. в области межфазной границы) концентрация $c_{B_{\ell} e q v}$ может быть значительно больше, чем в объеме фаз.

Легко показать, что такая оболочка приводит к торможению распада, если в ней понижается коэффициент диффузии компонента $A$, блокируя подвод вещества к растущему выделению. Однако, трудно ожидать реализации этого условия, если сформировавшаяся оболочка не является новой фазой.

Заметим, что качественно подобная оболочка возникает даже в случае $\tilde{v}=0$, но при наличии градиентного вклада в энергию смачивания $\left(\sigma_{A B}>0\right)$. Как видно из рис. 3, в случае $\sigma_{A B}>0$ межфазная граница обогащается компонентом $B$ (кривая 2), в то время как при $\sigma_{A B}=0$ концентрация компонента $B$ монотонно изменяется при переходе из одной фазы в другую (кривая $2^{\prime}$ ).

Таким образом, каждый из энергетических факторов, характеризующий склонность к распаду $(\tilde{v}<0)$ или „смачиванию“ межфазной границы $\left(\sigma_{A B}>0\right)$ может приводить к обогащению межфазной границы компонентом $B$. Формирование оболочки, исследованное ранее численным моделированием [16] для сплава $\mathrm{Fe}-\mathrm{Cu}-\mathrm{Ni}(\mathrm{Mn})$ было, по-видимому, обусловлено совместным действием этих факторов.

\section{4. Оболочка из чистого компонента. Пиннинг выделений}

Рассмотрим сплав, в котором имеются термодинамические стимулы к формированию в матрице выделе- ний обоих сортов, $A$ и $B$. Отметим, что эти стимулы исчезают при достаточно большом (по модулю) значении $\tilde{v}<0$, так как в этом случае тенденция распада по компонентам $A$ и $B$ сменяется на формирование фазы смешанного состава $A-B$ (см. обсуждение в [20]). Напротив, в случае $\tilde{v}>0$ стимулы распада по компонентам $A, B$ усиливаются. Приведенные далее результаты относятся к характерному сценарию, когда $\tilde{v}=0$, так что внутренняя энергия сплава имеет вид $g=\tilde{v}_{A M} c_{A}^{2}+\tilde{v}_{B M} c_{B}^{2}, \tilde{v}_{\alpha M}<0$. Сначала рассмотрим рост одиночного выделения $A$ из метастабильного пересыщенного твердого раствора, полагая что фигуративная точка сплава находится между бинодалью и спинодалью (по обоим компонентам). В процессе роста выделения $A$ компонент $B$ вытесняется из его объема в прилегающую область матрицы, где в некоторый момент зарождается выделение сорта $B$. Пусть $c_{A_{-} q q v}^{(M)}, c_{A_{e} q q v}^{(B)}-$ равновесные концентрации компонента $A$ в матрице и в объеме выделений $B$. Если до формирования выделения $B$ скорость роста выделения $A$, согласно (7), пропорциональна $c_{A_{-} q q v}^{(M)}$, то после возникновения выделения $B$ скорость подвода вещества к выделению $A$ определяется концентрацией $c_{A \text { eqv }}^{(B)}$. Если при этом энергия смешения $\tilde{v}_{B M}$ по абсолютной величине существенно превышает $\tilde{v}_{A M}$, между равновесными концентрациями устанавливается соотношение $c_{A_{-} e q v}^{(B)}<<c_{A_{-} e q v}^{(M)}$, что предотвращает дальнейший рост выделения $A$.
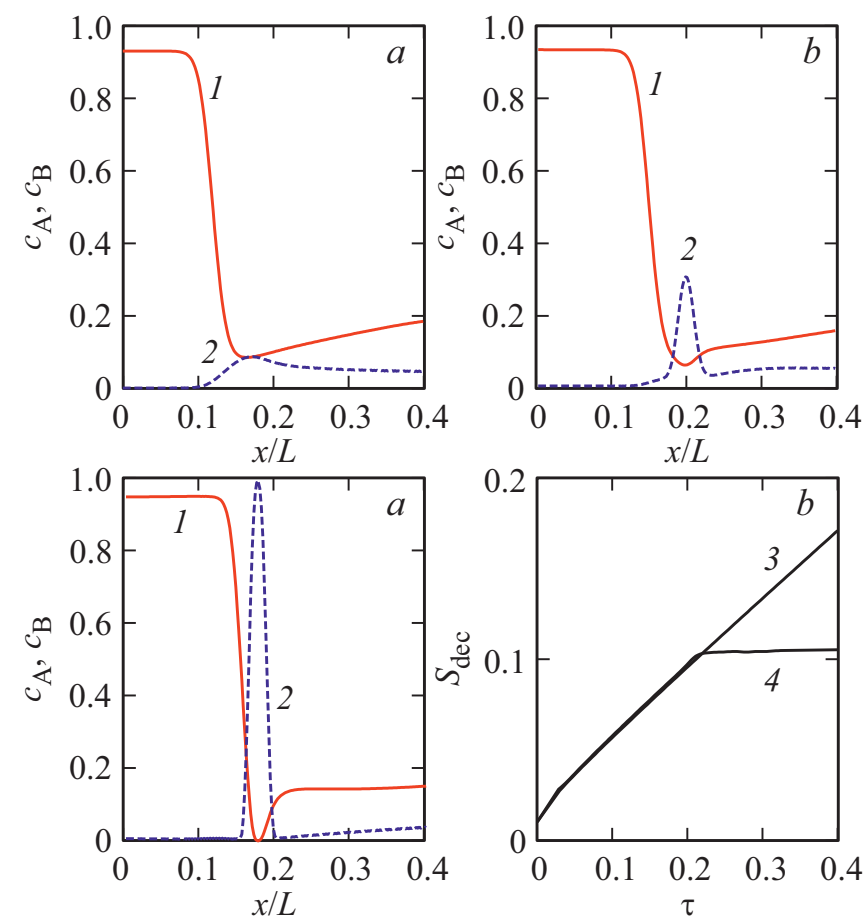

Рис. 4. Кинетика распада с формированием выделений $A(1)$ и $B(2)$, в моменты времени $\tau=0.11(a), 0.20(b), 0.25(c)$ и эволюция степени распада по компоненту $A(d)$ при $\tilde{v}_{B M}=0$ (3) и $\tilde{v}_{B M} / k T=-7(4) . D_{B} / D_{A}=1, \tilde{v}_{A M} / k T=-3$, $\tilde{v}_{B M} / k T=-7, \tilde{v}=0, \sigma_{A B}=0, \sigma_{A(B)}(R / L)^{2} / k T=4 \cdot 10^{-4}(\mathrm{eV})$, $c_{A}^{0}=0.2, c_{B}^{0}=0.05$. 


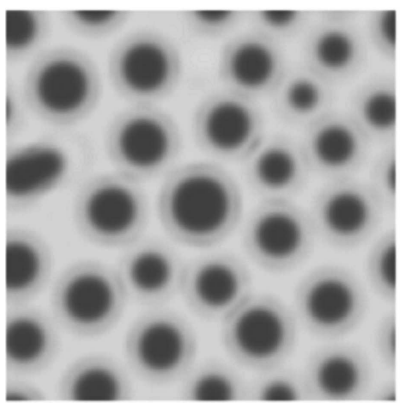

$\tau=0.17$

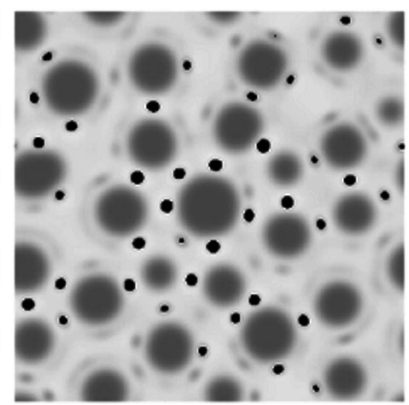

0.27

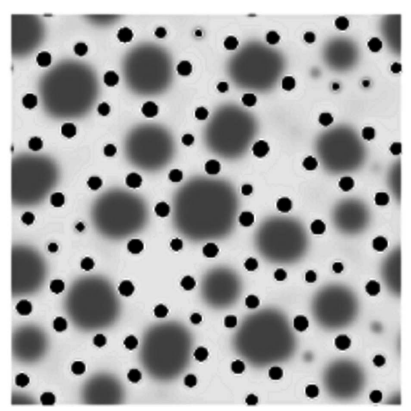

0.47

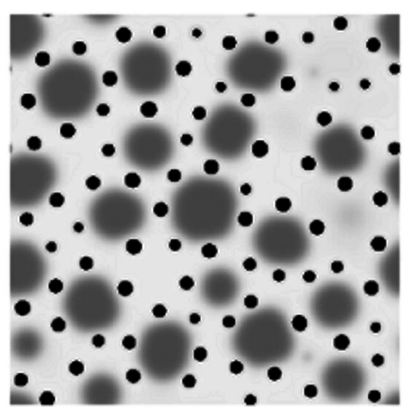

0.60

Рис. 5. Кинетика спинодального распада при $c_{A}^{0}=0.30, c_{B}^{0}=0.08, \tilde{v}_{A M} / k T=-3, \tilde{v}_{B M} / k T=-7, \tilde{v}=0, \sigma_{A(B)}(R / L)^{2} / k T=4 \cdot 10^{-4}$, $\sigma_{A B}=0, D_{B}^{(M)} / D_{A}=10^{-2}, D_{B}^{(A)} / D_{A}=1, D_{A}=$ const.

На рис. 4, $a, b$ представлены различные стадии рассматриваемого процесса: рост исходного выделения $A$ и образование выделения $B$. На рис. $4, d$ представлена эволюция степени распада для компонента $A$; можно видеть, что выделение $A$ монотонно растет в отсутствие выделения $B$, однако рост практически прекращается после формирования выделения $B$.

При повышении средних концентраций $c_{A}^{0}, c_{B}^{0}$, или при понижении температуры, в сплаве реализуется спинодальный распад (SD) по одному или обоим компонентам. При этом для формирования структуры выделений $A$ окруженных оболочкой $B$ благоприятна ситуация, когда $c_{A}^{0}$ лежит в спинодальной, а $c_{B}^{0}$ в метастабильной области диаграммы; тогда распад по компоненту $B$ происходит только в окрестности выделений $A$ после вытеснения из них компонента $B$, и не развивается в объеме матрице.

Рассмотренная выше задача с цилиндрической симметрией позволяет проанализировать условия формирования оболочки, однако не учитывает возможность развития распада вдоль межфазной границы. Поэтому далее мы рассматриваем решение уравнений (6) на 2D области в декартовых координатах, вводя в начальное состояние малые случайно распределенные гауссовы флуктуации состава и используя периодические граничные условия.

В отсутствие диффузии компонента $B\left(D_{B}=0\right)$ реализуется спинодальный распад по компоненту $A$ [22]. Кинетика распада в случае медленной диффузии компонента $B\left(D_{B}^{(M)} / D_{A} \ll 1\right)$ представлена на рис. 5. Выделения $A$ обозначены серым, выделения $B$ - черным, а матричная фаза белым цветом. Можно видеть, что вокруг выделений $A$ образуются цепочки из мелких выделений $B$. При этом прорастание выделения $A$ между соседними выделениями $B$ затруднено, поскольку сопровождается существенным повышением поверхностной энергии. В результате возникает ситуация качественно подобная пиннингу границ зерен мелкими выделениями. Заметим, что при высокой скорости диффузии компонента $B$ в матрице $\left(D_{B}^{(M)} / D_{A}>1\right)$, напротив, сначала
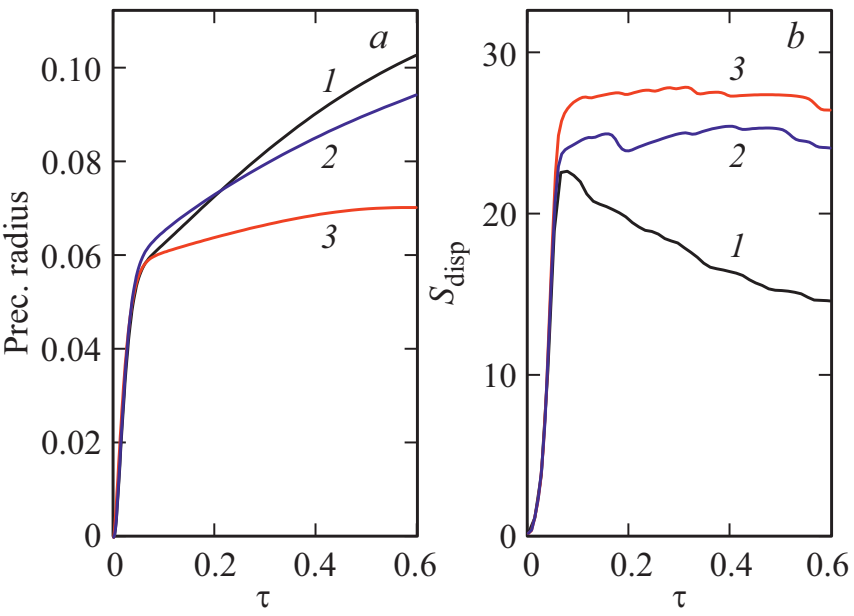

Рис. 6. Эволюция $(a)$ максимального размера выделения и $(b)$ степени дисперсности выделений при $D_{B}=0(1), D_{B}^{(M)}=10^{-2}$, $C_{B}^{(A)} / D_{A}=10^{-2}(2), D_{B}^{(M)} / D_{A}=10^{-2}, D_{B}^{(A)} / D_{A}=1$ (3). Остальные параметры те же, что и на рис. 5 .

реализуется распад по компоненту $B$, и возникающие затем выделения $A$ легко прорастают между хаотично распределенными выделениями $B$ [19].

На рис. 6 представлено изменение размера максимального выделения и степени дисперсности выделений при различном соотношении коэффициентов диффузии компонент $A$ и $B$. Видно, что наиболее эффективная стабилизация дисперсного состояния достигается, когда атомы сорта $B$ быстро диффундируют из объема выделений $A$, но теряют подвижность в матрице $\left(D_{B}^{(A)} / D_{A} \sim 1\right.$, $\left.D_{B}^{(M)} / D_{A} \ll 1\right)$.

Учет параметров $\tilde{v}, \sigma_{A B}$, ответственных за процессы смачивания, не изменяет качественно наблюдаемую картину (рис. 7). Выделения компонента $B$ располагаются в поверхностном слое выделений $A$, однако условие $\tilde{v}_{B M}<\tilde{v}_{A M}$, необходимое для торможения распада в обсуждаемом случае, приводит к формированию прерывистой, а не сплошной оболочки. При этом, если $\sigma_{A B}$ выше критического значения, поверхностная энергия (5) 


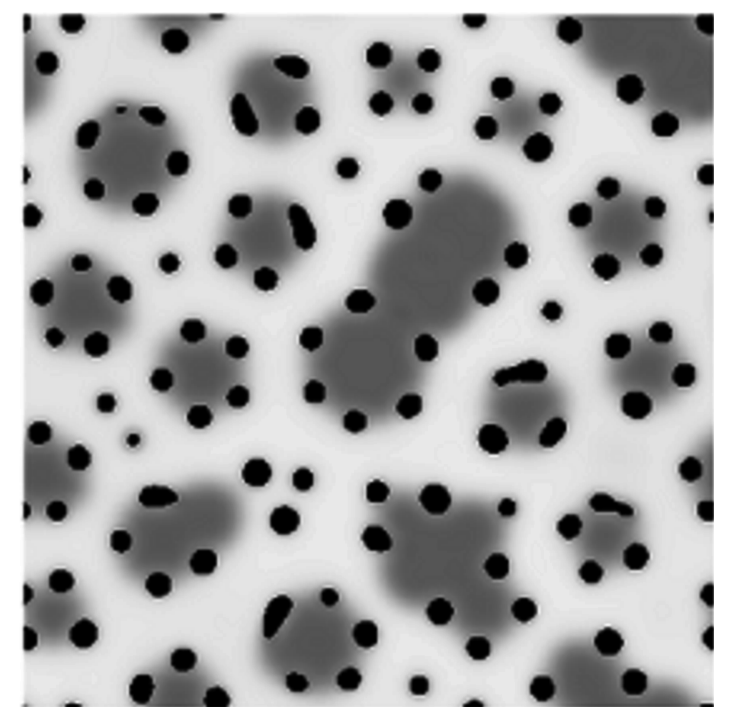

Рис. 7. Распределение выделений к моменту времени $\tau=0.60$ при $c_{B}^{0}=0.12, \tilde{v}=-3, \sigma_{A B}(R / L)^{2} / k T=2 \cdot 10^{-4}$. Остальные параметры те же, что и на рис. 5.

при контакте фаз становится отрицательной, и рассматриваемая модель теряет применимость.

Таким образом, совместное действие термодинамических и кинетических факторов, обеспечивающих образование частиц новой фазы на межфазной границе первичных выделений, может служить эффективным механизмом торможения распада, даже если сплошная оболочка вокруг выделений не формируется.

\section{5. Оболочка, образованная фазами промежуточного состава}

В общем случае энергии смешения $\tilde{v}_{\alpha \beta}$ в формуле (4) могут быть функциями концентраций, что может приводить к большому многообразию формирующихся микроструктур. При этом типичной ситуацией, как в бинарном, так и в трехкомпонентном сплаве, является формирование фазы промежуточного состава. Рассмотрим в качестве примера случай, когда в сплаве возможен распад по обоим компонентам $\left(\tilde{v}_{A M}<0, \tilde{v}_{B M}<0\right)$, причем энергия $\tilde{v}_{B M}$ зависит от концентраций

$$
\tilde{v}_{B M}=\tilde{v}_{B M}^{0}+\tilde{v}_{B M}^{(A)} c_{A}+\tilde{v}_{B M}^{(M)} c_{M}+\tilde{v}_{B M}^{(A M)} c_{A} c_{M}+\ldots
$$

Для формирования оболочки вокруг выделений $A$ необходимо обеспечить кооперативное развитие распада по компонентам $A$ и $B$. Это может быть обеспечено выбором $\tilde{v}_{B M}^{(A)}<0$, или $\tilde{v}_{B M}^{(M)}>0$, или $\tilde{v}_{B M}^{(A M)}<0$. Обратим внимание, что в последнем случае возникает дополнительный стимул распада по компоненту $B$ непосредственно на интерфейсе выделений $A$ (т. е. в области с промежуточными концентрациями обоих компонент $A, M)$.
Во всех этих случаях при определенном соотношении параметров вокруг выделений $A$ формируются выделения фазы промежуточного состава $(P I C)$. Если при этом компонент $B$ присутствует в сплаве в виде малой легирующей добавки, $c_{B}^{0} \ll c_{A}^{0}$, он может быть полностью израсходован на образование $P I C$, так что равновесное состояние сплава оказывается трехфазным: $M, A$ и мелкие выделения PIC. Задача о стабилизации выделений $A$ сводится к поиску таких условий распада, при которых PIC фаза образует ,запирающую оболочку“ вокруг выделений $A$. Наш анализ показывает, что оболочка наиболее выражена в случае $\tilde{v}_{B M}^{(A M)}<0$, когда PIC содержит все компоненты примерно в равных долях. Поэтому далее ограничимся обсуждением ситуации $\tilde{v}_{B M}=\tilde{v}_{B M}^{0}+\tilde{v}_{B M}^{(A M)} c_{A} c_{M}$, то есть не рассматриваем появление промежуточных фаз, обусловленных другими энергетическими параметрами, кроме $\tilde{v}_{B M}^{(A M)}<0$.

На рис. 8-10 представлена кинетика распада, описываемая уравнениями (6), и протекающая с образованием промежуточной фазы. Выделения компонента $A$ обозначены серым, матричная фаза белым, выделения PIC черным цветом. Морфология выделений в значительной степени определяется соотношением коэффициентов диффузии компонент. При $D_{A}^{(M)} \sim D_{B}^{(M)}$ и достаточной концентрации атомов обоих сортов, формируется микроструктура из цепочек чередующихся выделений $A$ и PIC (рис. 8); аналогичная картина наблюдалась ранее в работе [15] для спинодального распада в трехкомпонентном сплаве.

Если скорость диффузии компонента В в матрице существенно ниже, чем скорость диффузии компонента $A\left(D_{B}^{(M)} \ll D_{A}^{(M)}\right)$, формируется микроструктура из выделений компонента $A$ окруженных оболочкой $P I C$ (рис. 9). При этом для появления оболочки необходимо вытеснение компонента $B$ из объема растущих выделений $A$; поэтому при понижении коэффициента $D_{B}^{(A)}$ оболочка возникает на более поздних стадиях распада, когда скорость роста выделений становится уже достаточно низкой. Выделения, окруженные оболочкой формируются на промежуточной стадии распада и коагулируют на больших временах выдержки как за счет объединения частиц $P I C$, так и вследствие диффузии компонента $A$ через оболочку. Понижение средней концентрации $c_{B}^{0}$ приводит к ситуации, когда к моменту образования $P I C$ вблизи выделения недостаточно компонента $B$. В этом случае вокруг выделений $A$ возникает прерывистая оболочка, которая не является надежным стопором для развития распада (рис. 10).

Основное отличие рассмотренной ситуации от случая формирования оболочки из чистого компонента (раздел 4) состоит в следующем. Поскольку PIC содержит в значительном количестве компонент $A$, она в общем случае остается „прозрачной“ для диффузии этого компонента из объема матрицы к растущему выделению, и не тормозит рост выделений $A$. Однако, существенное замедление распада достигается, если коэффициенты 

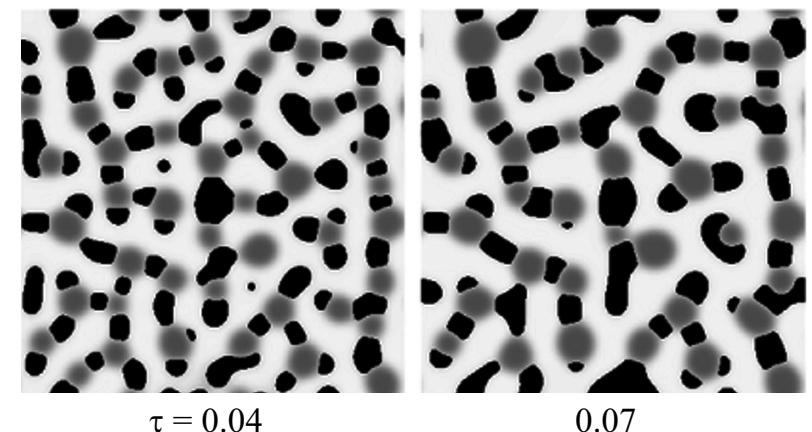

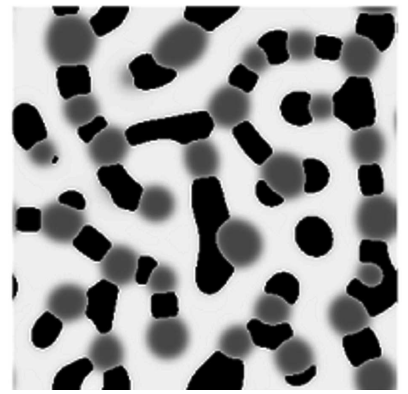

0.14

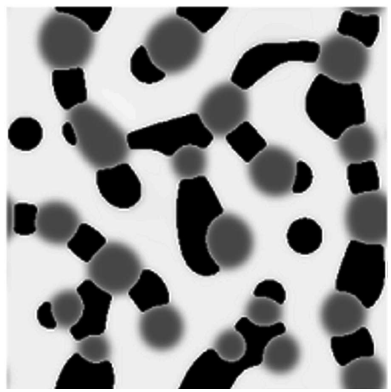

0.30

Рис. 8. Кинетика спинодального распада при $D_{A}^{(M)}=D_{B}, D_{A}^{(P I C)} / D_{A}^{(M)}=10^{-3} ; c_{A}^{0}=0.30, c_{B}^{0}=0.07, \tilde{v}_{A M} / k T=-3.5, \tilde{v}_{B M}^{0}=\tilde{v}=0$, $\tilde{v}_{B M}^{(A M)} / k T=-100, \sigma_{A(B)}(R / L)^{2} / k T=4 \cdot 10^{-4}, \sigma_{A B}=0$.

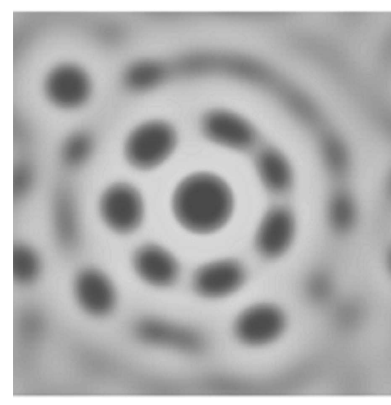

$\tau=0.04$

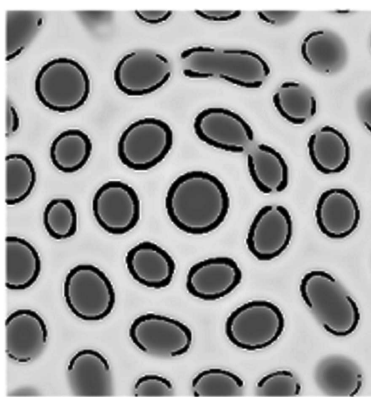

0.07

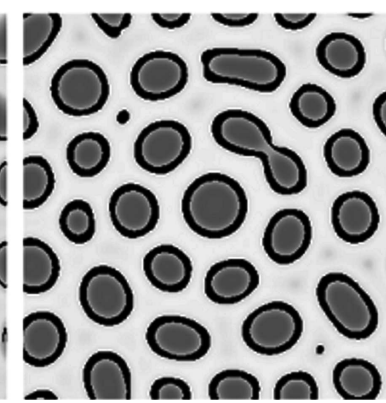

0.14

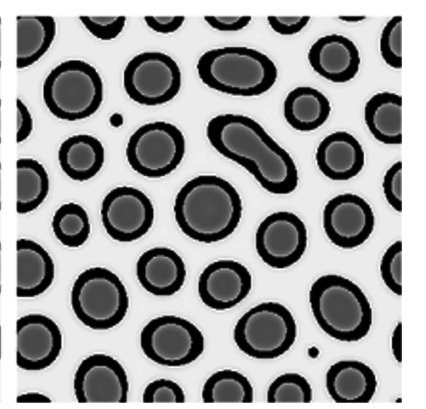

0.30

Рис. 9. Кинетика спинодального распада при $D_{B}^{(M, P I C)} / D_{A}^{(M)}=10^{-2}, D_{B}^{(A)} / D_{A}^{(M)}=3 \cdot 10^{-3}, D_{A}^{(P I C)} / D_{A}^{(M)}=10^{-3}$. Остальные параметры те же, что и на рис. 8.

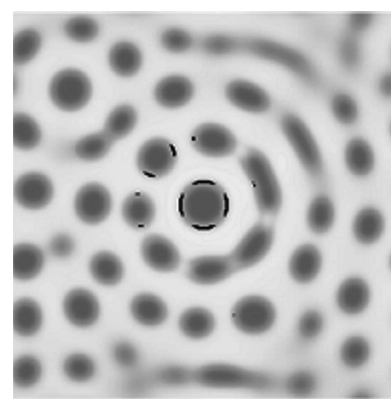

$\tau=0.02$

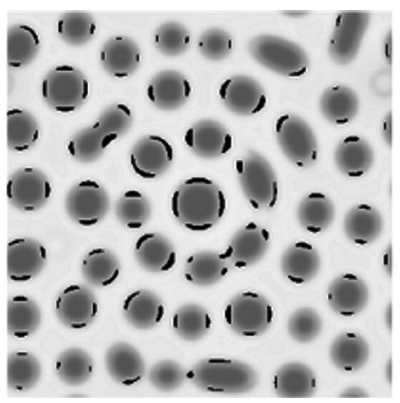

0.04

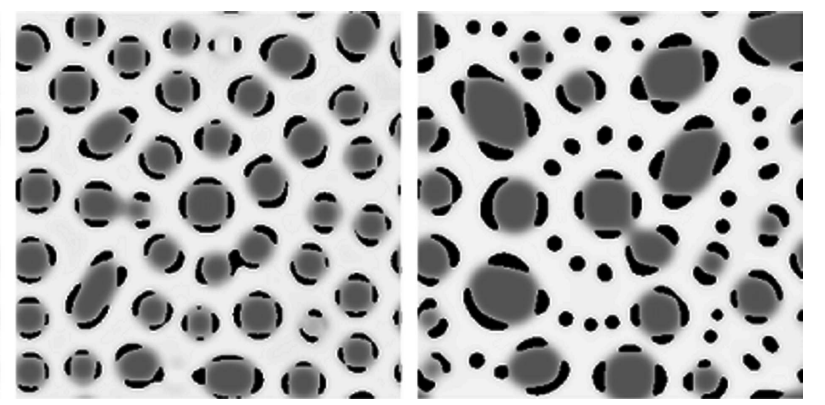

0.07
0.25

Рис. 10. Кинетика спинодального распада при $c_{B}^{0}=0.05, D_{B}^{(M, P I C)} / D_{A}=10^{-2}, D_{B}^{(A)} / D_{A}=1$. Остальные параметры те же, что и на рис. 8 .

диффузии компонент $A$ или $B$ в области фазы $P I C$ существенно ниже, чем коэффициент диффузии компонента $A$ в матрице; $D_{A}^{(P I C)} \ll D_{A}^{(M)}$ или $D_{B}^{(P I C)} \ll D_{A}^{(M)}$. В первом случае подвод компонента $A$ к выделению осуществляется через оболочку и затруднен, а во втором случае - рост выделения $A$ предполагает миграцию оболочки, обогащенной медленным компонентом $B$.

На рис. $11, a, b$ приведены изменение максимального размера выделения компонента $A$ и степени дисперсности, при различных соотношениях коэффициентов диффузии в фазах. Кривая 1 соответствует полностью замороженной диффузии компонента $B\left(D_{B}=0\right)$. В этом случае максимальный размер выделения монотонно возрастает, а степень дисперсности достигает максимума в конце волновой стадии спинодального распада, после чего монотонно снижается. Кривые 2 и 3 построены при различных значениях $D_{B}^{(M, A)}$, но в отсутствие понижения диффузионной подвижности компонента $A$ в области $P I C\left(D_{A}^{(P I C)}=D_{A}^{(M)}\right)$. Из сравнения кривых $1-3$ следует, что низкий коэффициент $D_{B}^{(M)}$ замедляет распад по компоненту $A$, так как способствует стабилизации оболочки.

Кривые 4 и 5 построены с учетом торможения диффузии компонента $A$ в $P I C\left(D_{A}^{(P I C)} \ll D_{A}^{(M)}\right)$, и отличаются только значением коэффициента диффузии $D_{B}^{(A)}$. Можно 

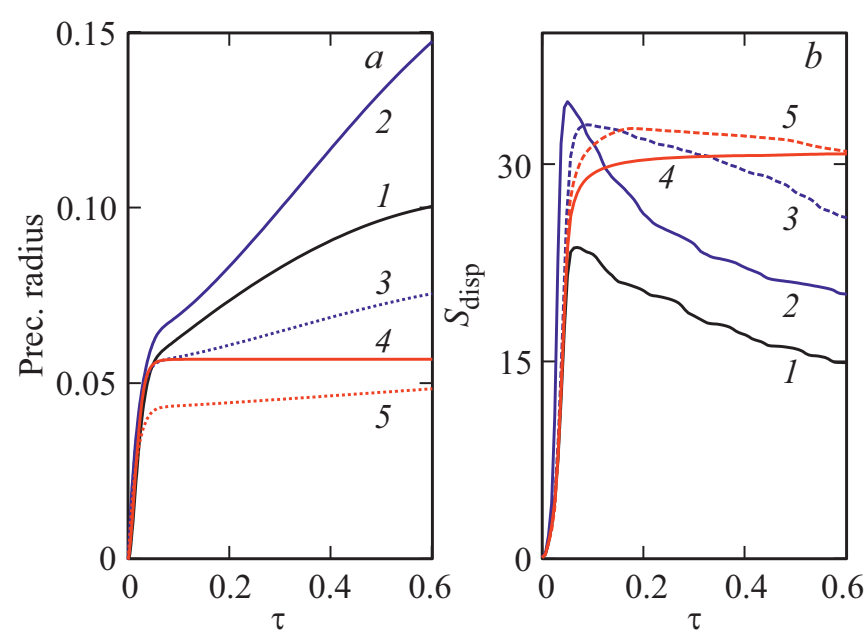

Рис. 11. Эволюция (a) максимального размера выделения и $(b)$ степени дисперсности выделений при (1) $D_{B}=0 ; \quad$ (2) $D_{B}^{(M, P I C)} / D_{A}^{(M)}=10^{-1}, D_{B}^{(A)} / D_{A}^{(M)}=1$, $D_{A}^{(P I C)} / D_{A}^{(M)}=1 ; \quad$ (3) $D_{B}^{(M, P I C)} / D_{A}^{(M)}=10^{-2}, \quad D_{B}^{(A)} / D_{A}^{(M)}=1$, $D_{A}^{(P I C)} / D_{A}^{(M)}=1$; (4) $D_{B}^{(M, P I C)} / D_{A}^{(M)}=10^{-2}, D_{B}^{(A)} / D_{A}^{(M)}=3 \cdot 10^{-3}$, $D_{A}^{(P I C)} / D_{A}^{(M)}=10^{-3} ; \quad(5) \quad D_{B}^{(M,(P I C))} / D_{A}^{(M)}=10^{-2}, D_{B}^{(A)} / D_{A}^{(M)}=1$, $D_{A}^{(P I C)} / D_{A}^{(M)}=10^{-3}$. Остальные параметры те же, что и на рис. 8 .

видеть, что высокий коэффициент $D_{B}^{(A)}$ эффективнее стабилизирует выделения $A$ на начальных стадиях, благодаря быстрому формированию оболочки, однако затем распад медленно продолжается вследствие разрушения оболочки. Наиболее эффективная стабилизация дисперсных выделений на стадии испарения-конденсации достигается для кривой 4 , которая соответствует случаю $D_{A}^{(P I C)} \ll D_{A}^{(M)}, D_{B}^{(M, A)} \ll D_{A}^{(M)}$ (рис. 9).

\section{6. Обсуждение результатов и выводы}

Исследована кинетика роста когерентных выделений из пересыщенного твердого раствора в трехкомпонентном сплаве. Показано, что эффективным механизмом торможения распада является формирование сплошной либо прерывистой „запирающей“ оболочки в виде прослойки новой фазы вокруг частиц выделений, в которой понижены растворимость или коэффициенты диффузии компонент сплава. В результате появления такой оболочки блокируется подвод вещества к растущему выделению, и следовательно достигается стабилизация дисперсного состояния.

Причиной появления оболочки могут быть кинетические (рис. 1) или термодинамические факторы (рис. 2-4,5), или их совместное действие (рис. 9, 10), в связи с чем свойства оболочки качественно различаются: в частности, может реализоваться равновесное обогащение межфазной границы примесью (рис. 2,3), выделение компонента $B$ в чистом виде (рис. $4,5,7)$ или фазы промежуточного состава на межфазной границе (рис. 9, 10). Формированию оболочки благоприятствует низкая скорость диффузии компонента $B$ в матрице, после его вытеснения из объема выделения, $B_{B}^{(M)} \ll D_{A}^{(M)}$, а также и в объеме выделений, $D_{B}^{(A)} \ll D_{A}^{(M)}$ (рис. 1, 5,9). При высокой скорости диффузии компонента $B$ возникают уединенные выделения этого компонента, либо ветвящаяся структура чередующихся выделений двух сортов (рис. 8).

Проведенный анализ показывает, что формирование оболочки вокруг выделений не всегда способствует замедлению кинетики распада. Так свойства кинетически обусловленной оболочки (рис. 1), обычно несущественно отличаются от объемных свойств сплава; она имеет непостоянную ширину и амплитуду изменения концентрации, и должна приводить к торможению распада главным образом на ранних стадиях.

Заметим, что торможение распада на стадии испарения-конденсации (Ostwald ripening) в результате формирования кинетически обусловленной оболочки наблюдалось в сплаве $\mathrm{Al}-\mathrm{Zr}-\mathrm{Sc}$ [14]. Этот сплав имеет специфичное соотношение коэффициентов диффузии компонент $D_{\mathrm{Sc}}^{M} \gg D_{\mathrm{Zn}}^{M}, D_{\mathrm{Sc}}^{P} \approx 0, D_{\mathrm{Zn}}^{P} \approx 0$, где $D_{\mathrm{Sc}(\mathrm{Zn})}^{M}$, $D_{\mathrm{Sc}(\mathrm{Zn})^{P}}-$ коэффициенты диффузии примесей $\mathrm{Sc}$ и $\mathrm{Zr}$ в матрице алюминия и в объеме выделений $\mathrm{Al}_{3} \mathrm{Sc}_{x} \mathrm{Zr}_{1-x}$ соответственно. На начальных стадиях распада условие $D_{\mathrm{Sc}}^{M} \gg D_{\mathrm{Sn}}^{M}$ обеспечивает рост выделений $\mathrm{Al}_{3} \mathrm{Sc}$ из пересыщенного твердого раствора, в отсутствие заметной диффузии Zr. Однако на стадии испарения-конденсации характерные времена кинетики распада оказываются сопоставимы со временами диффузии $\mathrm{Zr}$ в матрице, поэтому вокруг выделений $\mathrm{Al}_{3} \mathrm{Sc}$ формируется оболочка обогащенная $\mathrm{Zr}$. При этом условие $D_{\mathrm{Sc}}^{P} \approx 0$ предотвращает диффузию атомов Sc сквозь оболочку, а условие $D_{\mathrm{Zn}}^{P} \approx 0$ обеспечивает невозможность равномерного распределения атомов $\mathrm{Zr}$ по объему выделения. B результате обмен атомами Sc между преципитатами блокируется цирконием, так что дисперсное состояние сплава стабилизируется.

Оболочка, формирующаяся благодаря достижению локального равновесия в переходном слое на межфазной границе (рис. 2,3) наблюдалась экспериментально [17] и при численном моделировании с CALPHAD параметризацией $[16]$ в сплаве $\mathrm{Fe}-\mathrm{Cu}-\mathrm{Ni}(\mathrm{Mn})$. Она имеет ширину порядка межфазной границы и обычно небольшую амплитуду изменения концентрации. Моделирование методом Монте-Карло [18] показало, что такая оболочка не затормаживает распад, а наблюдаемое в эксперименте [17] повышение дисперсности выделений в сплаве $\mathrm{Fe}-\mathrm{Cu}-\mathrm{Ni}(\mathrm{Mn})$ обусловлено скорее ускоренным зарождением кластеров меди на примесных центрах. В тоже время, в рамках комбинированного подхода, включающего Монте-Карло и молекулярнодинамическое моделирование, показано [25], что формирование оболочки может приводить к торможению роста частиц в сплаве $\mathrm{Fe}-\mathrm{Cu}-\mathrm{Ni}-\mathrm{Al}$.

Весьма эффективное торможение роста частиц, выделяющихся из метастабильного пересыщенного твердого раствора реализуется в том случае, когда компонент $B$ имеет высокий стимул к распаду и выделяется 
на интерфейсе выделения $A$ в чистом виде, блокируя подвод вещества к выделению (рис. 4). При распаде в спинодальной области фазовой диаграммы для формирования такой оболочки необходимо дополнительное условие $D_{B} \ll D_{A}$, так как в противном случае в объеме сплава реализуется распад по компоненту $B$, еще до возникновения выделений $A$. При этом из результатов моделирования следует, что вплоть до весьма высоких концентраций $c_{B}^{0}$ вокруг выделений $A$ формируется не сплошная оболочка, а цепочки из мелких выделений компонента $B$, что, однако, достаточно для торможения распада (рис. 5,7). Отметим, что наблюдаемый эффект, имеет определенное сходство с явлением пиннинга границ зерен выделениями, предотвращающим развитие процесса рекристаллизации [26].

Другой механизм, обеспечивающий торможение роста выделений реализуется при низкой скорости диффузии компонента $A$ или $B$ через сплошную оболочку, $D_{A(B)}^{P I C} \ll D_{A}^{(M)}$ (рис. 9). Этот сценарий наиболее вероятен, если оболочка представляет собой новую фазу, в общем случае промежуточного состава. Оболочки, возникающие в результате распада с выделением новой фазы (разделы 4,5), расширяются до тех пор, пока не исчерпан лимит компонента $B$, и разрушаются на больших временах выдержки в силу коагуляции выделений образующей их фазы. Таким образом, их формирование не останавливает процесс распада, однако может существенно замедлить его кинетику (рис. 6, 11).

Полученные в работе выводы имеют достаточно общий характер и остаются справедливыми, несмотря на простоту модели и используемые приближения (континуальные уравнения диффузии в приближении среднего поля, когерентное сопряжение выделений, отсутствие упругого взаимодействия выделений). Хотя большинство представленных результатов касаются зарождения выделений по спинодальному механизму (рис. 5-11), выводы об условиях стабилизации дисперсного состояния за счет формирования оболочки вокруг выделений должны быть справедливыми и для других сценариев зарождения (флуктуационное, гетерогенное). Заметим, что в случае полукогерентных выделений обогащение межфазной границы примесью может реализоваться за счет различия энергий растворения примеси в объеме и на межфазной границе $[12,13]$. Возникающие в этом случае оболочки имеют ширину равную ширине межфазной границы, как и оболочки рассмотренные в разделе 3.2, но в отличие от последних, могут приводить к появлению равновесного размера выделений, при условии, что энергия сегрегации примеси на межфазной границе выше некоторого критического значения [12,13].

\section{Финансирование работы}

Работа выполнена в рамках государственного задания по темам „Магнит“ № А18-118020290129-5 и „Структура““ № А18-118020190116-6, а также при поддержке Российского научного фонда (грант № 18-12-00366)

\section{Конфликт интересов}

Авторы заявляют об отсутствии конфликта интересов.

\section{Список литературы}

[1] S. Jiang, H. Wang, Y. Wu, X. Liu, H. Chen, M. Yao, B. Gault, D. Ponge, D. Raabe, A. Hirata, M. Chen, Y. Wang, Z. Lu. Nature 544 (7651), 460 (2017).

[2] J. Millan, S. Sandlobes, A. Al-Zubi, T. Hickel, P. Choi, J. Neugebauer, D. Ponge, D. Raabe. Acta Mater. 76, 94 (2014).

[3] R.S. Rana, R. Purohit, S. Das. Int. J. Sci. Res. Publ. 2, 1 (2012).

[4] F. Sun, J.Y. Zhang, P. Vermaut, D. Choudhuri, T. Alam, S.A. Mantri, P. Svec, T. Gloriant, P.J. Jacques, R. Banerjee, F. Prima. Mater. Res. Lett. 5, 547 (2017).

[5] O. Gutfleisch. In: Nanoscale Magnetic Materials and Applications / Ed. J.P. Liu, E. Fullerton, O. Gutfleisch, D.J. Sellmyer. Springer, Berlin (2009).

[6] Z.B. Jiao, J.H. Luan, M.K. Miller, Y.W. Chung, C.T. Liu. Mater. Today 20, 3, 142 (2017).

[7] M.D. Mulholland, D.N. Seidman. Acta Mater. 59, 1881 (2011).

[8] L. Schemmann, S. Zaefferer, D. Raabe, F. Friedel, D. Mattissen. Acta Mater. 95, 386 (2015).

[9] M. Perez, F. Perrard, V. Massardier, X. Kleber, A. Deschamps, H. de Monestrol, P. Pareige, G. Covarel. Phil. Mag. 85, 2197 (2005).

[10] А.Г. Хачатурян. Теория фазовых превращений и структура твердых растворов. Наука, М. (1974), 384 с.

[11] T. Miyazaki. Mater. Transact. 43, 1266 (2002).

[12] И.К. Разумов. ФТТ 56, 749 (2014).

[13] S.B. Kadambi, S. Patala. Phys. Rev. Mater. 1, 043604 (2017).

[14] E. Clouet, L. Lae, T. Epicier, W. Lefebvre, M. Nastar, A. Deschamps. Nature Mater. 5, 482 (2006).

[15] L.Q. Chen. Computer simulation of spinodal decomposition in ternary systems. Acta Metallurg. Mater. 42, 10, 3503 (1994).

[16] T. Koyama, H. Onodera. Mat. Trans., 46, 6, 1187 (2005).

[17] M.K. Miller, B.D. Wirth, G.R. Odette. Mater. Sci. Eng. A 353, 133 (2003)

[18] Y. Wang, J. Yin, X. Liu, R. Wang, H. Hou, J. Wang. Prog. Natur. Sci.: Mater. Int. 27, 4, 460 (2017).

[19] S. Ghosh, A. Mukherjeez, T.A.Abinandanan, S. Bose. Phys. Chem. Chem. Phys. 19, 15424 (2017)

[20] M.S. Bhaskar, T.A. Abinandanan. Comput. Mater. Sci. 146, 73 (2018)

[21] Дж. Кристиан. Теория превращений в металлах и сплавах. Мир, М. (1978). 806 c.

[22] J.-F. Gouyet, M. Plapp, W. Dieterich, P. Maas. Adv. Phys. 52, 523 (2003).

[23] J.W. Cahn, J.E. Hilliard. J. Chem. Phys., 28, 258 (1958).

[24] Б.Б. Страумал. Фазовые переходы на границах зерен. Наука, М. (2003). 327 с.

[25] И.Н. Карькин, Л.Е. Карькина, Ю.Н. Горностырев, А.П. Коржавый. ФТТ 61, 724 (2019).

[26] T. Chakrabarti, S. Manna. Comp. Mater. Sci. 154, 84 (2018).

Редактор Т.Н. Василевская 